\title{
Implicit function and tangent cone theorems for singular inclusions and applications to nonlinear programming
}

\author{
Ewa Bednarczuk $^{1,2} \cdot$ Agnieszka Prusińska $^{3}$ - Alexey Tret'yakov Th, $^{1,4}$
}

Received: 22 January 2018 / Accepted: 14 October 2018 / Published online: 27 October 2018

(c) The Author(s) 2018

\begin{abstract}
The paper is devoted to the implicit function theorem involving singular mappings. We also discuss the form of the tangent cone to the solution set of the generalized equations in singular case and give some examples of applications to nonlinear programming and complementarity problems.
\end{abstract}

Keywords Implicit function theorem · Multifunction · Tangent cone · Singularity · Generalized equation $\cdot p$-Regularity

\section{Introduction}

The paper deals with so-called generalized equations, that is inclusions of the form

$$
0 \in f(x, y)+Q(y)
$$

where $f: X \times Y \mapsto Y^{*}, Y$ - Banach space, $Y^{*}$ dual space to $Y, X$ - normed space and $Q: Y \rightrightarrows Y^{*}$ is a set valued mapping.

Generalized equations provide a useful tool for the analysis of complementarity problems, first order optimality conditions in mathematical programming, equilibrium problems, and many aspects of nonlinear analysis. Recently metric regularity and Lipschizian stability of solutions to (1) have been studied in [1]. In [8] the generalized equation (1) has been accompanied by differential equation and is called differential generalized equation (DGE). The authors provided characterization of metric regular-

Agnieszka Prusińska

aprus@uph.edu.pl

1 Systems Research Institute, Polish Academy of Sciences, Newelska 6, 01-447 Warsaw, Poland

2 Faculty of Mathematics and Information Sciences, Warsaw University of Technology, Koszykowa 75, 00-662 Warsaw, Poland

3 Faculty of Science, Siedlce University, 3 Maja 54, 08-110 Siedlce, Poland

4 Dorodnitsyn Computing Center, Russian Academy of Sciences, Vavilova 40, Moscow, Russia 119991 
ity of control system coupled with a differential generalized equation, and characterize metric regularity of (DGE) in the finite dimensional case (see also [9,13,14,19] for other results related to existence and stability issues). For generalized equations of the form

$$
0 \in x-G(y)
$$

where $G: X \times Y \rightrightarrows Z$ and $X, Y, Z$ - Banach spaces the inverse mapping theorems and higher order metric regularity have been discussed in [10-12].

We investigate the problem (1) with the set valued mapping $Q$ of the form of the normal cone $Q=N_{C}(y)$, where $C \subset Y$ is a convex set, i.e.

$$
0 \in f(x, y)+N_{C}(y)
$$

see e.g. [17,24]. Here $N_{C}(y): Y \rightrightarrows Y^{*}$ is defined as

$$
N_{C}(y):= \begin{cases}\left\{z \in Y^{*}:\langle z, c-y\rangle \leq 0, \forall c \in C\right\}, & \text { if } y \in C \\ \emptyset, & \text { if } y \notin C .\end{cases}
$$

In the present paper we prove implicit function theorem for the problem (2). In [2], an implicit function theorem is provided for the problem $f(x, y) \in A$, where $f: X \times Y \mapsto Z$ is a differentiable mapping with $f_{y}^{\prime}$ being onto, $X$ topological space, $Y, Z$ - Banach spaces and $A \subset Z$, see also [16,22].

We propose another approach to implicit function theorem for generalized equation (2) that differs from the results of the above mentioned papers, i.e. we focus on singular mappings. We do not assume surjectivity of the derivative of the mapping $f$. Such problems (2) are called singular inclusion problems.

Let $\left(x_{0}, y_{0}\right)$ be the solution to the inclusion problem (2), i.e.

$$
0 \in f\left(x_{0}, y_{0}\right)+N_{C}\left(y_{0}\right) .
$$

We use $p$-regularity theory [7] in deriving our implicit function theorem for (2) which is an efficient tool to deal with differentiable mappings when the first derivative of $f$ at $\left(x_{0}, y_{0}\right)$ is not surjective (singular, degenerate) and so the strong regularity condition by Robinson (see [22]) is not applicable.

More precisely, we investigate the problem of existence of a locally defined mapping $\varphi: X \rightarrow Y, y=\varphi(x)$ which is a solution of (2) near a given solution $\left(x_{0}, y_{0}\right)$, that is $0 \in f(x, \varphi(x))+N_{C}(\varphi(x))$ for $x$ close to $x_{0}$ and $y_{0}=\varphi\left(x_{0}\right)$.

The classical implicit function theorem says that when a continuously differentiable function $f(x, y)$ vanishes at a point $\left(x_{0}, y_{0}\right)$ with $f_{y}^{\prime}\left(x_{0}, y_{0}\right)$ nonsingular (surjective), the equation $f(x, y)=0$ can be solved for $y$ in terms of $x$ in a neighborhood of $\left(x_{0}, y_{0}\right)$. This theorem has been extended in various directions, e.g. to Banach spaces $[25,26]$, to multivalued mappings $[2,10,11,16,22]$, to nonsmooth functions $[16,23]$, etc.

The results we present can be applied to parametric problems. There are numerous theorems concerning the solution existence of the problem with small parameter. Some 
of them deal with the problem of solution existence of the equation $f(x, y)=0$, where the mapping $f$ is singular, e.g. $[4,5,20,21,27]$. This analysis was based on the constructions of $p$-regularity theory that has been developed for the last forty years. The main constructions of this theory are described e.g. in [3-6] or in [25-27].

The organization of the paper is as follows. In Sect. 2 we formulate our main result which is Theorem 1 providing conditions for the existence of the implicit function $\varphi$ for the singular inclusion problem (2). In Sect. 3 we prove Lusternik-type theorem for (2).

\section{Implicit function theorem for singular inclusions}

Let $p \geq 2$ be a natural number and let $B: X^{p} \rightarrow Y$ be a continuous symmetric $p$-multilinear mapping. By $B[\cdot]^{p}: X \rightarrow Y$ we mean the $p$-form associated to $B$ and define it as follows

$$
B[x]^{p}:=B(x, x, \ldots, x), \quad x \in X .
$$

For any $h \in Y$ and $f \in \mathcal{C}^{p+1}\left(X \times Y, Y^{*}\right)$ we define a set-valued mapping $L_{h}: Y \rightrightarrows Y^{*}$,

$$
L_{h}(y):=\frac{1}{(p-1) !} f_{y}^{(p)}\left(x_{0}, y_{0}\right)[h]^{p-1}[h+y]+N_{C}(h+y) .
$$

Recall the Hausdorff distance between any sets $S_{1}$ and $S_{2}$,

$$
H\left(S_{1}, S_{2}\right):=\max \left\{\sup _{x \in S_{1}} \operatorname{dist}\left(x, S_{2}\right), \sup _{y \in S_{2}} \operatorname{dist}\left(y, S_{1}\right)\right\} .
$$

Without loss of generality assume that $x_{0}=0$ and $y_{0}=0$. Denote $U_{\gamma}(0)$, $V_{\gamma}(0)$ sufficiently small neighborhoods of 0 in $X$ and $Y$, respectively. Consider completely degenerate case up to the order $p$, that is assume that $f_{y}^{(k)}(0,0) \equiv 0$ for $k=1, \ldots, p-1$.

We prove the following theorem.

Theorem 1 Let $f \in \mathcal{C}^{p+1}\left(X \times Y, Y^{*}\right)$. Suppose that (4) is satisfied at $\left(x_{0}, y_{0}\right)=(0,0)$ and

$$
f_{y}^{(k)}(0,0)=0, \quad k=1,2, \ldots, p-1 .
$$

Assume the following conditions hold.

$1^{\circ}$ Banach condition:

for any $x \in U_{\gamma}(0)$, such that $f(x, 0) \neq 0$ and $\gamma>0$ is sufficiently small, there exists $h(x) \in Y, h \neq 0$ such that

$$
-f(x, 0) \in \frac{1}{(p-1) !} f_{y}^{(p)}(0,0)[h(x)]^{p}+N_{C}(h(x)),
$$


and $\|h(x)\| \leq c \cdot\|f(x, 0)\|^{1 / p}$, where $c>0$ is independent constant,

$2^{\circ}$ Strong $p$-regularity condition at the point 0 along $h=h(x), h \in Y$, i.e.

$$
H\left(L_{h}^{-1}\left(z_{1}\right), L_{h}^{-1}\left(z_{2}\right)\right) \leq \frac{c}{\|h\|^{p-1}}\left\|z_{1}-z_{2}\right\|, \quad \forall z_{1}, z_{2} \in Y^{*},
$$

$3^{\circ}$ p-factor approximation condition, i.e.

$$
\begin{aligned}
& \left\|f\left(x, y_{1}\right)-f\left(x, y_{2}\right)-\frac{1}{p !} f_{y}^{(p)}(0,0)\left[y_{1}\right]^{p}+\frac{1}{p !} f_{y}^{(p)}(0,0)\left[y_{2}\right]^{p}\right\| \\
& \quad \leq \delta\left(\left\|y_{1}\right\|^{p-1}+\left\|y_{2}\right\|^{p-1}\right)\left\|y_{1}-y_{2}\right\|
\end{aligned}
$$

for $x \in U_{\gamma}(0), y_{1}, y_{2} \in V_{\gamma}(0)$ and $\delta>0$ sufficiently small.

Then for sufficiently small $\varepsilon>0$ there exist a neighborhood $U_{\varepsilon}(0) \subset X$ and a mapping $\varphi(x): U_{\varepsilon}(0) \rightarrow Y$ such that for any $x \in U_{\varepsilon}(0)$ the mapping $\varphi(x)$ is a solution of the inclusion (2), i.e.

$$
0 \in f(x, \varphi(x))+N_{C}(\varphi(x))
$$

and

$$
\|\varphi(x)\| \leq m \cdot\|f(x, 0)\|^{1 / p},
$$

where $m>0$ is independent constant.

Remark In the case when $f(x, 0)=0$ we can take the mapping $\varphi(x)=0$ and Banach condition is trivial.

Before we prove this theorem we give two examples and for the convenience of the Reader we recall Robinson's strong regularity condition [22].

Definition 1 Let $f \in \mathcal{C}^{1}\left(X \times Y, Y^{*}\right)$ and $\left(x_{0}, y_{0}\right)$ be a solution of (2) and

$$
T y:=f\left(x_{0}, y_{0}\right)+f_{y}^{\prime}\left(x_{0}, y_{0}\right)\left(y-y_{0}\right)+N_{C}(y) .
$$

We say that (2) is strongly regular at $\left(x_{0}, y_{0}\right)$ with associated Lipschitz constant $\lambda$ if there exist neighborhoods $U$ of the origin in $Y^{*}$ and $V$ of $y_{0}$ such that the restriction to $U$ of $T^{-1} \cap V$ is a single valued function from $U$ to $V$ which is Lipschitzian on $U$ with modulus $\lambda$.

Example 1 Consider the nonlinear complementarity problem: to solve the system

$$
\begin{gathered}
f_{1}(x, y)=y_{1}^{2}-y_{2}^{2}-x_{1} \geq 0 \\
f_{2}(x, y)=y_{1} \cdot y_{2}-x_{2} \geq 0 \\
y \geq 0, \quad\langle f(x, y), y\rangle=0
\end{gathered}
$$

where

$$
x=\left(x_{1}, x_{2}\right)^{T} \in \mathbb{R}^{2}, \quad y=\left(y_{1}, y_{2}\right)^{T} \in \mathbb{R}_{+}^{2}, \quad f(x, y)=\left(f_{1}(x, y), f_{2}(x, y)\right)^{T},
$$


$f: \mathbb{R}^{2} \times \mathbb{R}_{+}^{2} \rightarrow \mathbb{R}^{2}$ and $x$ represents a small perturbation parameter. This problem is equivalent to the following generalized equation (see [22])

$$
0 \in f(x, y)+N_{\mathbb{R}_{+}^{2}}(y)
$$

and to analyze nonlinear complementarity problem (11) we can investigate inclusion (12) and apply Theorem 1 . It is obvious that the strong regularity condition (see Definition 1) fails at $(0,0)^{T}$ (since $f_{y}^{\prime}(0,0) \equiv 0$ ) and $T^{-1}$ is multivalued mapping, where $T y:=f_{y}^{\prime}(0,0) y+N_{\mathbb{R}_{+}^{2}}(y)$.

On the other hand, it turns out that all assumptions of Theorem 1 are fulfilled for $p=2$.

In this example the Banach condition $1^{\circ}$ takes the form

$$
-f(x, 0) \in f_{y}^{\prime \prime}(0,0)[h(x)]^{2}+N_{\mathbb{R}_{+}^{2}}\left(\left(h_{1}, h_{2}\right)\right)
$$

that is

$$
-\left(\begin{array}{l}
x_{1} \\
x_{2}
\end{array}\right) \in\left(\begin{array}{c}
2 h_{1}^{2}-2 h_{2}^{2} \\
2 h_{1} h_{2}
\end{array}\right)+N_{\mathbb{R}_{+}^{2}}\left(\left(h_{1}, h_{2}\right)\right)
$$

holds since $h_{1} \neq 0$ and $h_{2} \neq 0$ and hence $N_{\mathbb{R}_{+}^{2}}(h)=\{0\}$. This yields that the solution $h(x)$ of (13) can be found as a solution of the following equation

$$
-\left(\begin{array}{l}
x_{1} \\
x_{2}
\end{array}\right)=\left(\begin{array}{c}
2 h_{1}^{2}-2 h_{2}^{2} \\
2 h_{1} h_{2}
\end{array}\right)
$$

and we obtain $\|h(x)\| \leq c\|f(x, 0)\|^{1 / 2}, c>0$.

The second assumption, strong $p$-regularity $2^{\circ}$ holds since $f(x, y)$ is 2-regular (see e.g. in [6]) at $(0,0)^{T}$ with respect to $y$ along any $h \in Y$, such that $h_{1} \neq 0$ and $h_{2} \neq 0$, that is $\operatorname{Im} f^{\prime \prime}(0,0) h=\mathbb{R}^{2}$ and $N_{\mathbb{R}_{+}^{2}}(\cdot)=\{0\}$.

The $p$-factor approximation condition $3^{\circ}$ is immediately satisfied due to the form of the mapping $f(x, y)$. This means that all conditions of Theorem 1 are fulfilled and therefore for small $x$ there exists a mapping $\varphi(x)$ which acts from a neighborhood of $0 \in \mathbb{R}^{2}$ into $\mathbb{R}^{2}$ and for which (10) is satisfied.

In the next example we illustrate the application of Theorem 1 to the standard nonlinear programming problem

$$
\begin{array}{r}
\min \xi(y) \\
\text { subject to } g(y) \leq 0,
\end{array}
$$

where $g=\left(g_{1}, \ldots, g_{m}\right)^{T}, \mathcal{L}(y, \lambda)=\xi(y)+\langle\lambda, g(y)\rangle, \lambda=\left(\lambda_{1}, \ldots, \lambda_{m}\right)^{T}$. The Karush-Kuhn-Tucker optimality conditions (KKT) for (14) are

$$
\mathcal{L}_{y}^{\prime}=0, g(y) \leq 0, \lambda \geq 0,\langle\lambda, g(y)\rangle=0
$$


This conditions can be written as the generalized equation

$$
0 \in\left(\begin{array}{c}
\mathcal{L}_{y}^{\prime}(y, \lambda) \\
-g(y)
\end{array}\right)+N_{\mathbb{R}^{n} \times \mathbb{R}_{+}^{m}}\left(\begin{array}{l}
y \\
\lambda
\end{array}\right)
$$

and we verify the assumptions of the Theorem 1 at the solution $\left(y_{0}, \lambda_{0}\right)$ for (16).

Example 2 Consider the following nonlinear programming problem

$$
\min y_{1}^{4}-y_{2}^{4}-x y_{1}
$$

subject to

$$
\begin{aligned}
& y_{1}^{3}-2 y_{2}^{3} \leq 0 \\
& y_{1}^{3}+2 y_{2}^{3} \leq 0
\end{aligned}
$$

where $x$ represents a small perturbation parameter. For $x_{0}=0$ a solution of (17) is $y_{0}=0$ and $\mathcal{L}(y, \lambda, x)=y_{1}^{4}-y_{2}^{4}-x y_{1}+\lambda_{1}\left(y_{1}^{3}-2 y_{2}^{3}\right)+\lambda_{2}\left(y_{1}^{3}+2 y_{2}^{3}\right)$.

Then Karush-Kuhn-Tucker optimality conditions are as follows

$$
\begin{aligned}
& 4 y_{1}^{3}-x+3 \lambda_{1} y_{1}^{3}+3 \lambda_{2} y_{1}^{2}=0 \\
& -4 y_{2}^{3}-6 \lambda_{1} y_{2}^{2}+6 \lambda_{2} y_{2}^{2}=0 \\
& y_{1}^{3}-2 y_{2}^{3} \leq 0, \quad y_{1}^{3}+2 y_{2}^{3} \leq 0, \quad\langle\lambda, g(y)\rangle=0
\end{aligned}
$$

where

$$
\begin{aligned}
\lambda & =\left(\lambda_{1}, \lambda_{2}\right)^{T}, \quad y=\left(y_{1}, y_{2}\right)^{T}, g(y)=\left(g_{1}(y), g_{2}(y)\right)^{T}, \\
g_{1}(y) & =y_{1}^{3}-2 y_{2}^{3}, \quad g_{2}(y)=-y_{1}^{3}+2 y_{2}^{3} .
\end{aligned}
$$

Consider the case $\lambda_{0}=(0,0)^{T}$. Generalized equation (2) for the problem (17) is

$$
0 \in\left(\begin{array}{c}
4 y_{1}^{3}-x+3 \lambda_{1} y_{1}^{2}+3 \lambda_{2} y_{1}^{2} \\
-4 y_{2}^{3}-6 \lambda_{1} y_{2}^{2}+6 \lambda_{2} y_{2}^{2} \\
y_{1}^{3}-2 y_{2}^{3} \\
y_{1}^{3}+2 y_{2}^{3}
\end{array}\right)+N_{\mathbb{R}^{2} \times \mathbb{R}_{+}^{2}}\left(\begin{array}{c}
y_{1} \\
y_{2} \\
\lambda_{1} \\
\lambda_{2}
\end{array}\right)
$$

It is obvious that (18) is not strong regular in the sense by Robinson at the solution point $y_{0}=(0,0)^{T}, \lambda_{0}=(0,0)^{T}, x_{0}=0$. However, one can verify that all assumptions of Theorem 1 are fulfilled for $p=3$ with $h=\left(h_{y_{1}}, h_{y_{2}}, 0, h_{\lambda_{2}}\right)$, since in this case we can take $h_{y_{1}} \neq 0, h_{y_{2}} \neq 0, h_{\lambda_{2}} \neq 0$ for $x \neq 0$ and normal cone operator $N_{\mathbb{R}^{2} \times \mathbb{R}_{+}^{2}}\left(\begin{array}{l}y_{1} \\ y_{2} \\ \lambda_{1} \\ \lambda_{2}\end{array}\right)$ has the following form $\left(\begin{array}{c}0 \\ 0 \\ -\alpha \\ 0\end{array}\right), \alpha \in \mathbb{R}_{+}$. 
This means that for small perturbation $x$ there exists a mapping $\varphi(x)=$ $(y(x), \lambda(x))^{T}$ such that inclusion (18) (or (16)) holds and hence KKT conditons (15) holds as well and the estimation for $\varphi(x)$ is as follows

$$
\|\varphi(x)\|=\|y(x)\|+\|\lambda(x)\| \leq m \cdot\left\|\mathcal{L}_{y}^{\prime}(0,0, x)\right\|^{1 / 3} \leq \bar{m}\|x\|^{1 / 3}
$$

where $m>0, \bar{m}>0$ are independent constants.

The following theorem is essential in the proof of Theorem 1 (see [15]).

Theorem 2 (Contraction multimapping principle, CMP) Let $Z$ be a complete metric space with distance $\rho$ and $z_{0} \in Z$. Assume that we are given a multimapping

$$
\Phi: U_{\varepsilon}\left(z_{0}\right) \rightrightarrows Z
$$

on a ball $U_{\varepsilon}\left(z_{0}\right)=\left\{z: \rho\left(z, z_{0}\right)<\varepsilon\right\} \quad(\varepsilon>0)$ where the sets $\Phi(z)$ are non-empty and closed for any $z \in U_{\varepsilon}\left(z_{0}\right)$. Further, assume that there exists a number $\theta, 0<\theta<1$ such that

1. $H\left(\Phi\left(z_{1}\right), \Phi\left(z_{2}\right)\right) \leq \theta \rho\left(z_{1}, z_{2}\right)$ for any $z_{1}, z_{2} \in U_{\varepsilon}\left(z_{0}\right)$

2. $\rho\left(z_{0}, \Phi\left(z_{0}\right)\right)<(1-\theta) \varepsilon$.

Then, for every number $\varepsilon_{1}$ which satisfies the inequality

$$
\rho\left(z_{0}, \Phi\left(z_{0}\right)\right)<\varepsilon_{1}<(1-\theta) \varepsilon
$$

there exists $z \in B_{\varepsilon_{1} /(1-\theta)}\left(z_{0}\right)=\left\{\omega: \rho\left(\omega, z_{0}\right) \leq \varepsilon_{1} /(1-\theta)\right\}$ such that

$$
z \in \Phi(z)
$$

Proof of Theorem 1 Suppose that $x \in U_{\gamma}(0), y_{1}, y_{2} \in V_{\gamma}(0)$, for sufficiently small $\gamma>0$. Moreover, assume that there exists $h(x) \in Y, h \neq 0$ such that Banach condition holds true.

Let us define a mapping $r: U_{\gamma}(0) \times Y \rightarrow W_{\varepsilon}(0)$, where $\varepsilon>0$ is sufficiently small and $W_{\varepsilon}(0) \subset Y^{*}$, as follows

$$
r(x, h+y):=\frac{1}{(p-1) !} f_{y}^{(p)}(0,0)[h]^{p-1}[h+y]-f(x, h+y) .
$$

Now, for $y=0$

$$
L_{h}(0)=\frac{1}{(p-1) !} f_{y}^{(p)}(0,0)[h]^{p}+N_{C}(h) .
$$

Then, using a right inverse of $L_{h}$ we obtain

$$
0 \in L_{h}^{-1}\left(\frac{1}{(p-1) !} f_{y}^{(p)}(0,0)[h]^{p}+N_{C}(h)\right) .
$$


Introduce an auxiliary mapping $\Phi: U_{\gamma}(0) \times V_{\gamma}(0) \rightarrow Y$,

$$
\Phi(x, y):=L_{h}^{-1}(r(x, h+y)) .
$$

We show that there exists $y=y(x) \in \Phi(x, y(x))$ or, in other words,

$0 \in N_{C}(h+y(x))+f(x, h+y(x))$. For this purpose we check the assumptions of CMP.

From the assumptions $2^{\circ}$ and $3^{\circ}$ of Theorem 1 we obtain

1. $H\left(\Phi\left(x, y_{1}\right), \Phi\left(x, y_{2}\right)\right)^{2^{\circ}} \leq \frac{c}{\|h\|^{p-1}}\left\|r\left(x, h+y_{1}\right)-r\left(x, h+y_{2}\right)\right\|=\frac{c}{\|h\|^{p-1}}$.

$$
\begin{aligned}
& \cdot\left\|\frac{1}{(p-1) !} f_{y}^{(p)}(0,0)[h]^{p-1}\left[y_{1}-y_{2}\right]-f\left(x, h+y_{1}\right)+f\left(x, h+y_{2}\right)\right\| \\
& \stackrel{3^{\circ}}{\leq} \frac{c \cdot \delta\left(\|h\|^{p-1}+\|h\|^{p-1}\right)}{\|h\|^{p-1}}\left\|y_{1}-y_{2}\right\| \leq 2 c \cdot \delta\left\|y_{1}-y_{2}\right\| .
\end{aligned}
$$

Moreover, since $0 \in L_{h}^{-1}(-f(x, 0))$ then

2. $H(\Phi(x, 0), 0) \leq H\left(L_{h}^{-1}(r(x, h)), L_{h}^{-1}(-f(x, 0))\right) \leq$

$$
\begin{aligned}
& \stackrel{2^{\circ}}{\leq} \frac{c}{\|h\|^{p-1}}\|r(x, h)+f(x, 0)\| \\
& =\frac{c}{\|h\|^{p-1}}\left\|\frac{1}{(p-1) !} f_{y}^{(p)}(0,0)[h]^{p}-f(x, h)+f(x, 0)\right\| \\
& \stackrel{3^{\circ}}{\leq} \frac{c \cdot \delta}{\|h\|^{p-1}}\|h\|^{p-1}\|h\| \leq c \delta\|h\| .
\end{aligned}
$$

It means that all assumptions of CMP hold. Hence there exists $y=y(x)$ such that $y(x) \in L_{h}^{-1}(r(x, h+y(x)))$, or in other words

$$
0 \in f(x, h+y(x))+N_{C}(h+y(x)) .
$$

Let $\varphi(x):=h+y(x)$. We finish the proof of the Theorem 1 by obtaining the following estimation

$$
\|y(x)\|=o\left(\|f(x, 0)\|^{1 / p}\right)
$$

and hence

$$
\|\varphi(x)\| \leq m\|f(x, 0)\|^{1 / p},
$$

where $m>0$ independent constant.

\section{P-order tangent cone theorem for singular inclusions: generalization of Lusternik theorem}

Consider the following mapping

$$
F(x):=f(x)+N_{C}(x)
$$

and the generalized equation

$$
0 \in F(x)
$$


where $f: X \rightarrow X^{*}$ and is sufficiently smooth, $X-$ Banach space, $C$ is a nonempty closed convex set in $X$ and $N_{C}(x)$ is defined in (3). Let $x_{0}$ be the solution to inclusion (24), i.e. $0 \in F\left(x_{0}\right)$ and introduce the tangent cone to the set

$$
M_{F}\left(x_{0}\right):=\left\{z \in X: 0 \in f(z)+N_{C}(z)\right\}
$$

at the point $x_{0}$.

Definition 2 We say that $h$ belongs to the tangent cone $T M_{F}\left(x_{0}\right)$ of the set $M_{F}\left(x_{0}\right)$ at the point $x_{0}$ if $\forall t \in[0, \varepsilon)$, where $\varepsilon>0$ is sufficiently small, there exists a mapping $w: X \rightarrow X$ such that

$$
0 \in f\left(x_{0}+t h+w(t h)\right)+N_{C}\left(x_{0}+t h+w(t h)\right)
$$

and $\|w(t h)\|=o(t)$.

It is enough to consider the completely degenerate case up to the order $p$, i.e. the case where $f^{(k)}\left(x_{0}\right)=0, k=1, \ldots, p-1, p \geq 2$.

For any $h \in X$ we define a set-valued mapping $L_{h}: X \rightrightarrows X^{*}$,

$$
L_{h}(x):=\frac{1}{(p-1) !} f^{(p)}\left(x_{0}\right)[h]^{p-1}[h+x]+N_{C}\left(x_{0}+h+x\right) .
$$

We can describe the tangent cone $T M_{F}\left(x_{0}\right)$ by means of the following theorem, which generalizes Lusternik theorem for singular inclusions (cf. [9,18]).

Theorem 3 Let $f: X \rightarrow X^{*}, f \in \mathcal{C}^{p+1}(X), f$ be completely degenerate at $x_{0}$ up to the order $p$ and $0 \in F\left(x_{0}\right)$. Assume moreover, that $\bar{h} \in \operatorname{Ker}^{p} L_{h}(0)$, i.e.

$$
0 \in L_{t \bar{h}}(0) \Leftrightarrow 0 \in f^{(p)}\left(x_{0}\right)[t \bar{h}]^{p}+N_{C}\left(x_{0}+t \bar{h}\right) \quad \forall t \in[0, \varepsilon)
$$

where $\varepsilon>0$ is sufficiently small and for the mapping $F(x)$ strong p-regularity condition holds along $\bar{h}$ at the point $x_{0}$, that is

$$
H\left(L_{t \bar{h}}^{-1}\left(y_{1}\right), L_{t \bar{h}}^{-1}\left(y_{2}\right)\right) \leq \frac{c}{t^{p-1}}\left\|y_{1}-y_{2}\right\|
$$

where $y_{1}, y_{2} \in X^{*}$ and $t \in[0, \varepsilon)$.

Then

$$
\bar{h} \in T M_{F}\left(x_{0}\right) .
$$

Proof For the sake of simplicity we assume that $x_{0}=0$. Let us define $r(t \bar{h}+x):=\frac{1}{(p-1) !} f^{(p)}(0)[t \bar{h}]^{p-1}[t \bar{h}+x]-f(t \bar{h}+x)$.

Then

$$
0 \in L_{t \bar{h}}^{-1}\left(\frac{1}{(p-1) !} f^{(p)}(0)[t \bar{h}]^{p}+N_{C}(t \bar{h})\right) .
$$

Consider the following mapping

$$
\Phi(x):=L_{t \bar{h}}^{-1}(r(t \bar{h}+x)) .
$$


We show that there exists $w(t \bar{h})$ such that $w(t \bar{h}) \in \Phi(w(t \bar{h}))$ and $\|w(t \bar{h})\|=o(t)$ or, in other words $0 \in f(t \bar{h}+w(t \bar{h}))+N_{C}(t \bar{h}+w(t \bar{h})$. From the condition (26) for $\left\|y_{1}\right\| \leq \alpha t,\left\|y_{2}\right\| \leq \alpha t$ where $\alpha>0$ is sufficiently small we have

$$
\begin{aligned}
1^{\circ} & H\left(\Phi\left(y_{1}\right), \Phi\left(y_{2}\right)\right)=H\left(L_{t \bar{h}}^{-1}\left(r\left(t \bar{h}+y_{1}\right)\right), L_{t \bar{h}}^{-1}\left(r\left(t \bar{h}+y_{2}\right)\right)\right) \\
& \leq \frac{c}{t^{p-1}}\left\|\frac{1}{(p-1) !} f^{(p)}(0)[t \bar{h}]^{p-1}\left(y_{1}-y_{2}\right)-f\left(t \bar{h}+y_{1}\right)+f\left(t \bar{h}+y_{2}\right)\right\| \\
& \leq \delta(t)\left\|y_{1}-y_{2}\right\|, \text { where } \delta(t) \rightarrow 0 \text { while } t \rightarrow 0 . \\
2^{\circ} & H(\Phi(0), 0) \leq \\
& \leq H\left(L_{t \bar{h}}^{-1}(r(t \bar{h})), L_{t \bar{h}}^{-1}\left(\frac{1}{(p-1) !} f^{(p)}(0)[t \bar{h}]^{p}+N_{C}(t \bar{h})\right)\right) \\
& \leq \frac{c}{t^{p-1}}\left\|r(t \bar{h})-\frac{1}{(p-1) !} f^{(p)}(0)[t \bar{h}]^{p}+N_{C}(t \bar{h})\right\| \leq \\
& \leq \frac{c}{t^{p-1}}\left\|\frac{1}{(p-1) !} f^{(p)}(0)[t \bar{h}]^{p}-f(t \bar{h})+0\right\| \leq c_{1} t^{2} .
\end{aligned}
$$

It means that all conditions of CMP are fulfilled and hence there exists $w(t \bar{h}) \in \Phi(t \bar{h})$ and it follows that $w(t \bar{h}) \in L_{t \bar{h}}^{-1}(r(t \bar{h}+w(t \bar{h})))$, or in other words $0 \in f(t \bar{h}+w(t \bar{h}))+N_{C}(t \bar{h}+w(t \bar{h}) \forall t \in[0, \varepsilon)$ and $\|w(t \bar{h})\|=o(t)$, i.e. $\bar{h} \in T M_{F}\left(x_{0}\right)$.

Example 3 Let $F(x)=f(x)+N_{C}(x)$, where $f(x)=\left(f_{1}(x), f_{2}(x)\right)^{T}, x \in \mathbb{R}^{2}$, $f_{1}(x)=x_{2}^{2}-x_{1}^{2}, f_{2}(x)=x_{1} x_{2}, x \geq 0, C=\mathbb{R}_{+}^{2}, x_{0}=0$. Here $p=2$, $N_{\mathbb{R}_{+}^{2}}(0)=\left\{z \in \mathbb{R}^{2}:\langle z, \xi\rangle \leq 0, \forall \xi \in \mathbb{R}_{+}^{2}\right\}$ and the generalized equation is

$$
0 \in\left(\begin{array}{c}
x_{2}^{2}-x_{1}^{2} \\
x_{1} x_{2}
\end{array}\right)+N_{\mathbb{R}_{+}^{2}}\left(\begin{array}{l}
x_{1} \\
x_{2}
\end{array}\right) .
$$

Taking $\bar{h}=\left(\bar{h}_{x_{1}}, \bar{h}_{x_{2}}\right)^{T}$ where $\bar{h}_{x_{1}}=0 \bar{h}_{x_{2}}=1$ we have $N_{\mathbb{R}_{+}^{2}}\left(\begin{array}{l}0 \\ 1\end{array}\right)=\alpha\left(\begin{array}{c}-1 \\ 0\end{array}\right)$, $\alpha>0$ and $\bar{h} \in \operatorname{Ker}^{2} L_{h}(0)$ if $\left(\begin{array}{c}\bar{h}_{x_{1}} \\ 0\end{array}\right)-\left(\begin{array}{c}\alpha \\ 0\end{array}\right)=0$, i.e. $\bar{h}_{x_{1}}^{2}=\alpha$ and $\bar{h}_{x_{1}}= \pm \sqrt{\alpha}$ and all the assumptions of Theorem 3 are fulfilled. It means that $\bar{h}=(0,1)^{T} \in T M_{F}(0)$.

Both theorems presented in the present paper have been proved only for completely degenerate case but they could be easily generalized for non-complete degeneration. Such generalization follows directly from the construction of the $p$-factor operator and we refer the Reader to the papers devoted to the $p$-regularity theory (see e.g. $[6,7,21,26])$.

Acknowledgements The results of the research of the second and third author carried out under the research theme No. 165/00/S were financed from the science grant granted by the Ministry of Science and Higher Education. The work of the third author was supported also by the Russian Foundation for Basic Research (project No. 17-07-00510, 17-07-00493) and the RAS Presidium Programme (programme 27).

Open Access This article is distributed under the terms of the Creative Commons Attribution 4.0 International License (http://creativecommons.org/licenses/by/4.0/), which permits unrestricted use, distribution, and reproduction in any medium, provided you give appropriate credit to the original author(s) and the source, provide a link to the Creative Commons license, and indicate if changes were made. 


\section{References}

1. Artacho, F.J.A., Mordukhovich, B.S.: Metric regularity and Lipschitzian stability of parametric variational systems. Nonlinear Anal. Theory Methods Appl. 72, 1149-1170 (2010)

2. Avakov, E.R., Magaril-Il'yaev, G.G.: An implicit-function theorem for inclusions. Math. Notes 91(6), 764-769 (2012)

3. Brezhneva, O.A., Tret'yakov, A.A.: Optimality conditions for degenerate extremum problems with equality constraints. SIAM J. Control Optim. 42(2), 729-745 (2003)

4. Brezhneva, O.A., Evtushenko, YuG, Tret'yakov, A.A.: The 2-factor-method with a modified lagrange function for degenerate constrained optimization problems. Dokl. Math. 73(3), 384-387 (2006)

5. Brezhneva, O.A., Evtushenko, YuG, Tret'yakov, A.A.: The p-regularity theory and Poincaré's small parameter method. Dokl. Math. 74(3), 869-873 (2006)

6. Brezhneva, O.A., Tret'yakov, A.A.: Implicit function theorems for nonregular mappings in Banach spaces. Exit from singularity. In: Banach spaces and their applications in analysis. De Gruyter Proceedings in Mathematics, pp. 285-302 (2007)

7. Brezhneva, O.A., Tret'yakov, A.A., Marsden, J.E.: Higher-order implicit function theorems and degenerate nonlinear boundary-value problems. Commun. Pure Appl. Anal. 7(2), 293-315 (2008)

8. Cibulka, R., Dontchev, A.L., Krastanov, M., Veliov, V.M.: Metrically regular differential generalized equations. SIAM J. Control Optim. 56(1), 316-342 (2018)

9. Dontchev, A.L., Rockafellar, R.T.: Implicit Functions and Solution Mappings. Springer, Berlin (2009)

10. Frankowska, H.: High order inverse function theorems. Annales de 1'I.H.P., Sect. C tome S6, 283-303 (1989)

11. Frankowska, H.: Some inverse mapping theorems. Ann. Inst. Henri Poincarè 7(3), 183-234 (1990)

12. Frankowska, H., Quincampoix, M.: Holder metric regularity of set-valued maps. Math. Program. Ser. A 132, 333-354 (2012)

13. Gwinner, J.: On differential variational inequalities and projected dynamical systems-equivalence and a stability result. Discrete Contin. Dyn. Syst. Supl. 2007, 467-476 (2007)

14. Gwinner, J.: On a new class of differential variational inequalities and a stability result. Math. Program. 139, 205-221 (2013)

15. Ioffe, A.D., Tikhomirov, V.M.: Theory of Extremal Problems. Nauka, Moscow, (1974). English transl, North-Holland, Amsterdam (1979)

16. Izmailov, A.F.: Strongly regular nonsmooth generalized equations. Math. Program. Ser. A B 147(1-2), 581-590 (2014)

17. Klatte, D., Kummer, B.: Nonsmooth Equations in Optimization. Kluver Academic Publisher, Dordrecht (2002)

18. Ledzewicz, U., Schättler, H.: A high-order generalization of the Lyustenik theorem. Nonlinear Anal. 34, 793-815 (1998)

19. Pang, J.-S., Stewart, D.E.: Differential variational inequalities. Math. Program. Ser. A 113, 345-424 (2008)

20. Prusińska, A., Tret'yakov, A.: A remak on the existence of solutions to nonlinear equations with degenerate mappings. Set-Valued Anal. 16, 93-104 (2008)

21. Prusińska, A., Tret'yakov, A.: On the existence of solutions to nonlinear equations involving singular mappings with non-zero p-kernel. Set-Valued Anal. 19, 399-416 (2011)

22. Robinson, S.M.: Strongly regular generalized equations. Math. Oper. Res. 5, 43-62 (1980)

23. Robinson, S.M.: An implicit-function theorem for a class of nonsmooth functions. Math. Oper. Res. 16, 292-309 (1981)

24. Rockafellar, R.T., Wets, R.J.-B.: Variational Analysis, vol. 317. Springer, Berlin (1998)

25. Tret'yakov, A.A.: The implicit function theorem in degenerate problems. Russ. Math. Surv. 42, 179180 (1987)

26. Tret'yakov, A.A., Marsden, J.E.: Factor analysis of nonlinear mappings: p-regularity theory. Commun. Pure Appl. Anal. 2(4), 425-445 (2003)

27. Tret'yakov, A.A.: On solution existence for the perturbed degenerate optimization problem with equality constraints: p-order stability condition. Dokl. Math. 81, 354-357 (2010) 